\title{
Suzaku detection of a highly carbon enriched plasma in $\mathrm{BD}+30^{\circ} 3639$
}

\author{
M. Kokubun ${ }^{1}$, M. Murashima ${ }^{1}$, K. Makishima ${ }^{1,2}$, K. Matsushita ${ }^{3}$, \\ J. Kotoku ${ }^{4}$, H. Murakami ${ }^{5}$, K. Hayashida ${ }^{6}$, H. Matsumoto ${ }^{7}$, \\ K. Hamaguchi ${ }^{8}$, and K. Arnaud ${ }^{8}$ \\ ${ }^{1}$ Department of Physics, University of Tokyo, Bunkyo, Tokyo, 113-0033, Japan; \\ email: kokubun@phys.s.u-tokyo.ac.jp \\ ${ }^{2}$ Cosmic Radiation Laboratory, RIKEN, Wako, Saitama, 351-0198, Japan \\ ${ }^{3}$ Department of Physics, Tokyo University of Science, Shinjuku, Tokyo, 162-8601, Japan \\ ${ }^{4}$ Department of Physics, Tokyo Institute of Technology, Meguro, Tokyo, 152-8551, Japan \\ ${ }^{5}$ ISAS/JAXA, Sagamihara, Kanagawa, 229-8510, Japan \\ ${ }^{6}$ Department of Space and Earth Science, Osaka University, Toyonaka, Osaka, 560-0043, Japan \\ ${ }^{7}$ Department of Physics, Kyoto University, Sakyo-ku, Kyoto, 608-8502, Japan \\ ${ }^{8}$ NASA/GSFC, Greenbelt, MD 20771, USA
}

\begin{abstract}
We have observed the X-ray brightest planetary nebula $\mathrm{BD}+30^{\circ} 3639$ with the new Japanese astronomical satellite Suzaku. With its superior spectral resolution back-illuminated CCD camera, a blend of K-lines from highly ionized C, N, O elements are successfully resolved into individual species, and an extremely high relative abundance ratio of $\mathrm{C} / \mathrm{O} \sim 40$ is obtained. This strongly suggests the direct measurement of the helium shell-burning products.
\end{abstract}

Keywords. nuclear reactions, nucleosynthesis, abundances, stars: mass loss, planetary nebulae: individual $\left(\mathrm{BD}+30^{\circ} 3639\right)$, X-rays: individual $\left(\mathrm{BD}+30^{\circ} 3639\right)$,

\section{Introduction}

The origin and mechanism of the nucleosynthesis of heavy elements, especially carbon, has been the subject of fundamental astrophysical studies. Intermediate mass stars whose mass is smaller than 8 solar masses are considered to synthesize $\mathrm{C}, \mathrm{O}$, and $\mathrm{Ne}$ via helium burning, and the central star itself transforms into a planetary nebula $(\mathrm{PN})$ at the final evolutionary stage, in which synthesized metals are widely spread into the interstellar medium (ISM). Therefore, PNe are the most suitable objects to investigate nucleosynthesis, if the observer can extract products at the specific stage. X-ray emissions from $\mathrm{PNe}$ have been detected from several sources, and are thought to be from hot plasmas heated by shocks of fast stellar winds (Kwok 1982). Since the fast stellar winds evolve at the later evolutionary stages, the X-ray observations can be a useful tool for the investigation of the specific phase of the nucleosynthesis. However, past X-ray observations have failed to estimate the abundance ratios of $\mathrm{C}, \mathrm{N}$, and $\mathrm{O}$, since the energy resolution of $\mathrm{X}$-ray CCD cameras are not enough to resolve a blend of K-lines.

The 5th Japanese X-ray satellite Suzaku was successfully launched on 2005 July 10. The X-ray Imaging Spectrometer (XIS) consists of four CCD cameras located at the focus point of four X-ray Telescopes (XRT), and one of them, named XIS-1, utilizes the back-illuminated (BI) technique which enables a higher sensitivity at the lower energy side below $1 \mathrm{keV}$ than canonical front-illuminated CCD chips. The energy resolution of XIS-BI is $\sim 50 \mathrm{eV}$ (FWHM) at $0.5 \mathrm{keV}$ (Hayashida et al. 2004), and the energy response 
for a monochromatic line shows much smaller low-energy tails than those used in past $\mathrm{X}$-ray missions. Thus we have observed the $\mathrm{BD}+30^{\circ} 3639$ with $S u z a k u$, to obtain the first detection of individual K-lines from the highly ionized elements of major helium burning products.

\section{Observation and Results}

We have selected the X-ray brightest $\mathrm{PN}, \mathrm{BD}+30^{\circ} 3639$, as the first target of observation with Suzaku. Many past X-ray observations on this target have been performed (Kreysing et al. 1992, Kastner et al. 2000, Arnaud et al. 1996, Maness et al. 2003). The X-ray emitting region is known to be spatially extended with an angular diameter of $\sim 4^{\prime \prime}$, and located inside the optical shell. A strong $\mathrm{K}$ emission line from Ne IX is detected at $0.9 \mathrm{keV}$ together with a large spectral hump below $0.7 \mathrm{keV}$, which suggests the existence of highly non-solar abundance plasma.

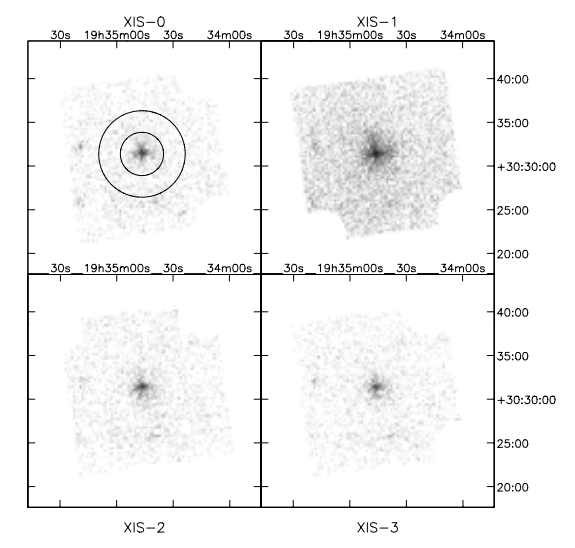

Figure 1. The raw $0.3-7 \mathrm{keV}$ X-ray image of $\mathrm{BD}+30^{\circ} 3639$ obtained with the Suzaku XIS- $0,1,2$, and 3 . Two circles indicate the on-source and background region.

The Suzaku observation was performed on 2005 September 20 during the initial performance verification phase of the satellite, with a total exposure time of $34.4 \mathrm{ksec}$. As shown in Figure 1, the PN is successfully detected with all four XIS, as a point source with the moderate spatial resolution of XRT. The source spectrum was extracted from a 2.5 ' radius circle, while the background was obtained from the surrounding annulus with a radius of 5 '.

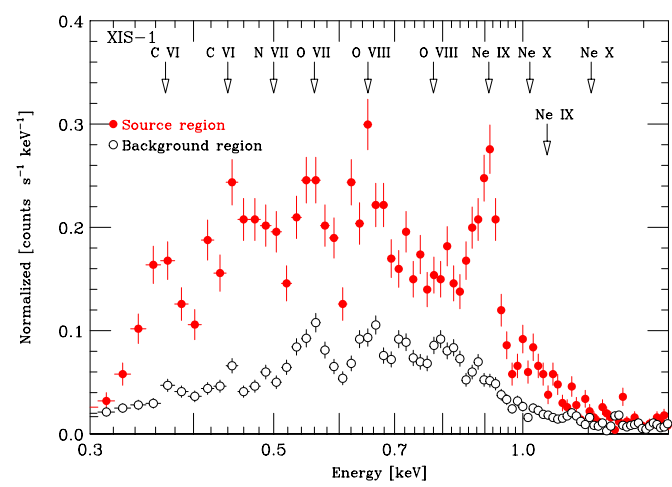

Figure 2. The $0.3-1.5 \mathrm{keV}$ spectrum of $\mathrm{BD}+30^{\circ} 3639$ obtained with XIS-BI. 
We have applied standard data processing and screening criteria, which is the most reliable at this early stage of Suzaku, and also applied the self-calibrating process for the energy gain and excess absorption due to the contaminant in front of the CCD camera, by use of this observation itself and the in-orbit calibration data. The resultant spectra obtained from the source and background regions are shown in Figure 2. The $\mathrm{K}_{\alpha}$ line from hydrogen-like $\mathrm{C}$ VI is for the first time separately detected at $0.37 \mathrm{keV}$, together with other K lines from O VII, O VIII, and Ne IX. Since the C VI to O VII line ratio from solar abundance plasma at $k T \sim 0.2 \mathrm{keV}$ is expected to be much smaller $(\sim 0.02)$ than the observed value, this clear detection strongly suggests a highly carbon enriched plasma.

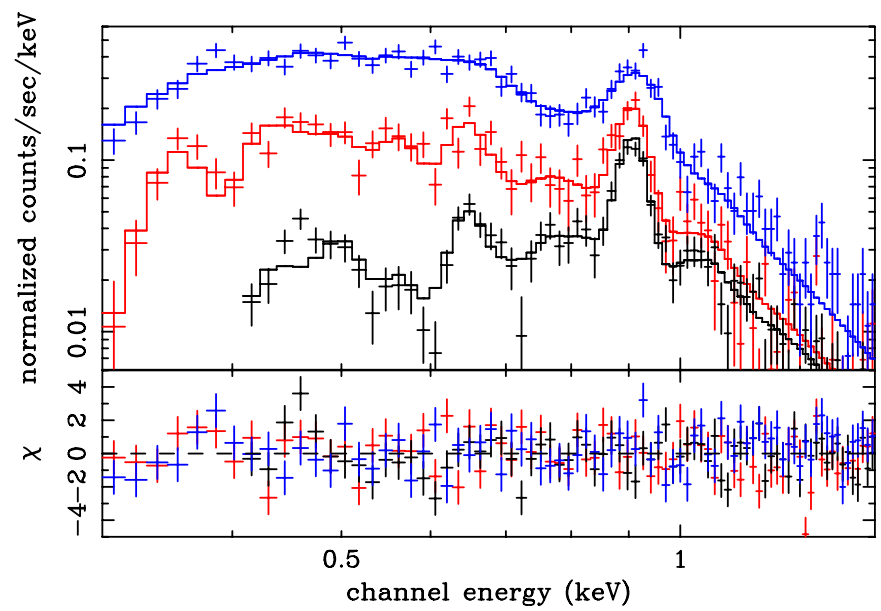

Figure 3. The background-subtracted XIS-BI (middle), XIS-FI (bottom), and Chandra ACIS-S (top) spectra of $\mathrm{BD}+30^{\circ} 3639$, simultaneously fitted by an absorbed vAPEC model with nonsolar abundances.

In the spectral fitting procedure, we included the spectrum obtained with Chandra ACIS-S to reduce the systematic uncertainties. We applied a single temperature vAPEC model, leaving the abundances of $\mathrm{C}, \mathrm{N}, \mathrm{O}, \mathrm{Ne}$, and Fe free. The He abundance was fixed to solar, and other heavy elements were neglected. As shown in figure 3, this model brought us a reasonable joint fit to all spectra with $\chi^{2} / \nu=331 / 228$, at the plasma temperature of $k T=0.19 \pm 0.01 \mathrm{keV}$ and absorption with a column density of $\mathrm{N}_{\mathrm{H}}=2.1_{-0.7}^{+0.4} \times 10^{21} \mathrm{~cm}^{-2}$.

Although we have successfully obtained the abundances of each element, the absolute metal abundances cannot be reliable because of the fixed abundance of helium, and a large contribution from metals to the continuum. On the other hand, the relative ratios among metals are expected to be well constrained since individual lines are clearly resolved thanks to the superior energy resolution of Suzaku-XIS. As shown in figure 4, we produced a confidence contour for the carbon and oxygen abundances, and confirmed that they are constrained in a narrow region of $\mathrm{C} / \mathrm{O}=85_{-14}^{+19}$ solar at $90 \%$ confidence. The ratios of $\mathrm{N} / \mathrm{O}, \mathrm{Ne} / \mathrm{O}$ are obtained in a same manner as $3.2_{-2.3}^{+2.3}$ and $5.8_{-0.9}^{+1.7}$, respectively.

\section{Discussion}

With the fine energy resolution of XIS, Suzaku has for the first time revealed an extremely carbon enriched abundance. From optical or infrared observations on this $\mathrm{PN}$, the nebular abundance ratios have been obtained as $\mathrm{C} / \mathrm{O} \sim 3.7, \mathrm{~N} / \mathrm{O} \sim 1.8$, and $\mathrm{Ne} / \mathrm{O} \sim 2.8$ (Bernard-Salas et al. 2003). When compared with these values, it is infered 


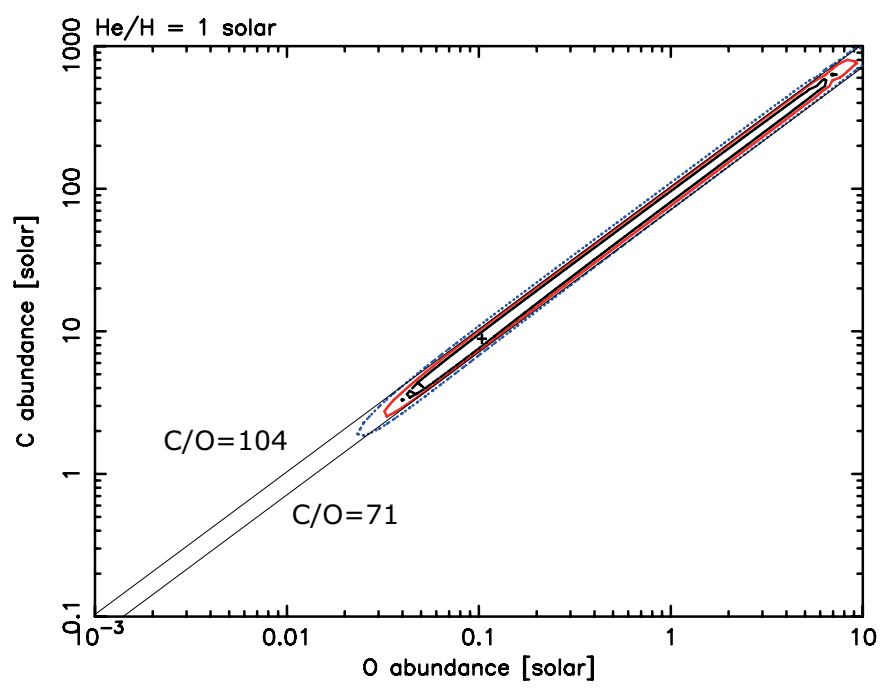

Figure 4. Confidence contour $(68,90$, and $99 \%)$ of the $\mathrm{C}$ against $\mathrm{O}$ abundance.

that the X-ray emitting plasma represent the abundance of an inner stellar nucleosynthesis zone, which is also supported by the image obtained with Chandra.

The resultant high carbon-to-oxygen number ratio of $\sim 40$ is in fact a typical value in the He burning layer for an initial mass of $\sim 2 M_{\odot}$, which is derived as a competition between the triple- $\alpha$ and ${ }^{12} \mathrm{C}(\alpha, \gamma){ }^{16} \mathrm{O}$ reactions (Suda et al. 2004). The $\mathrm{N} / \mathrm{O}$ number ratio of $\sim 0.42$ can be expressed as a remainder of the CNO cycle, and the high $\mathrm{Ne} / \mathrm{O}$ number ratio can be produced via the chain reaction of ${ }^{14} \mathrm{~N}(\alpha, \gamma){ }^{18} \mathrm{~F}\left(\beta^{+}, \nu\right)^{18} \mathrm{O}(\alpha, \gamma){ }^{22} \mathrm{Ne}$, which is also proceeding during the He shell flashes. Therefore, the hydrogen envelope of the $\mathrm{PN}$ has been possibly vented, and a mixture of the convective and radiative zones of the helium layer ejected, wind-driven, and heated up as X-ray emitting plasma. Thus, the observational results with Suzaku lead us to the conclusion that we have successfully detected the site of carbon synthesis via He burning at the final evolutionary stage of the stellar nucleosynthesis.

\section{Acknowledgements}

This research was partially supported by the Ministry of Education, Science, Sports and Culture, Grant-in-Aid for Young Scientist (A), 17684006, 2005.

\section{References}

Arnaud, K., Borkowsk, K.J., \& Harrington, J.P. 1996, ApJ 462, 75

Bernard-Salas, J., Pottasch, S. R., Wesselius, P. R., \& Feibelman, W. A. 2003, A $\& A, 406,165$

Hayashida, K., et al. 2004, SPIE, 5488, 73

Kastner, J.H., Soker, N., Vrtilek, S.D., \& Dgani, R. 2000, ApJ, 545, 57

Kreysing, H.C., Diesch, C., Zweigle, J., et al. 1992, A\&A, 264, 623

Kwok, S. 1982, ApJ, 258, 280

Maness, H.L., Vrtilek, S.D., Kastner, J.H., \& Soker, N. 2003, ApJ, 589, 439

Murashima, M. 2006, PhD thesis, University of Tokyo.

Suda, T., Arikawa, M., Machida, M.N., Fujimoto, M.Y., \& Iben, I.J. 2004, ApJ, 611, 476 